\title{
Desenvolvimento de Instrumento em Doenças Raras: Acesso à Saúde e ao Suporte Social
}

\section{Development of Instrument in Rare Diseases: Access to Health and Social Support \\ Desarrollo de Instrumento en Enfermedad Raras: Acceso a la Salud y al Apoyo Social}

\author{
Isabel Cristina de Barros Salviano ${ }^{1}$ \\ Universidade Salgado de Oliveira (Universo) \\ Martha Moreira Cavalcante Castro \\ Universidade Federal da Bahia (UFBA) \\ Marcos Antônio Almeida Matos \\ Carolina Villa Nova Aguiar \\ Escola Bahiana de Medicina e Saúde Pública
}

\begin{abstract}
Resumo
Introdução: A condição crônica degenerativa relacionada às Doenças Raras (DR) é permeada por processos sociais e de acesso à saúde complexos, cuja compreensão exige avaliação na perspectiva dos acometidos. Objetivo: Construir um instrumento que possibilite avaliar a percepção do acesso à saúde e ao suporte social destinado aos acometidos por DR. Métodos: Estudo de desenvolvimento de um instrumento embasado no modelo proposto por Luiz Pasquali. Resultados: Após análise fatorial, o resultado final que mostrou evidências preliminares de validação foi um instrumento com quatro domínios: Recursos de Saúde, Recursos Financeiros, Aspectos Sociais e Aspectos Individuais, com 10 itens. Considerações finais: O instrumento final desenvolvido reuniu evidências de validade, revelando qualidade dos itens em representar os conceitos subjacentes a cada domínio. Por meio de suas propriedades psicométricas, o instrumento mostrou que tem representatividade no que concerne à percepção do acometido por DR quanto ao seu acesso à saúde e ao suporte social.

Descritores: doenças raras, apoio social, acesso aos serviços de saúde, estudos de validação
\end{abstract}

\section{Abstract}

Introduction: The chronic degenerative condition related to Rare Diseases (DR) is permeated by complex social processes and access to health, whose understanding requires evaluation from the perspective of affected patients. Objective: To build up an instrument that allows those affected by these pathologies to evaluate their perception regarding access to healthcare and social support. Methods: Study on the development of an instrument based on the model proposed by Luiz Pasquali. Results: After a factorial analysis, the final result that showed preliminary evidence of validation was an instrument with four domains: Health Resources, Financial Resources, Social Aspects, and Individual Aspects, with 10 items. Final considerations: The final instrument developed gathered evidence of validity, revealing item quality in representing the concepts underlying each domain. Through its psychometric properties, the instrument showed that it has representativeness regarding the perception of the affected by DR regarding their access to health and social support..

Descriptors: rare diseases, social support, health services accessibility, validation studies

\section{Resumen}

Introducción: La condición crónica degenerativa relacionada a las Enfermedades Raras (ER) está impregnada por procesos sociales y de acceso a la salud complejos, cuya comprensión exige evaluación en la perspectiva de los acometidos. Objetivo: Construir un instrumento que posibilite evaluar la percepción del acceso a la salud y al apoyo social destinado a los acometidos por ER. Métodos: Estudio sobre el desarrollo de un instrumento en el modelo propuesto por Luiz Pasquali. Resultados: Después del análisis factorial, el resultado final que mostró evidencias preliminares de validación fue un instrumento con cuatro dominios: Recursos de Salud, Recursos Financieros, Aspectos Sociales y Aspectos Individuales, con 10 ítems. Consideraciones finales: El instrumento final desarrollado reunió evidencia de validez, revelando la calidad del elemento al representar los

\footnotetext{
${ }^{1}$ Endereço de contato: Av. Dom João VI, 275, Brotas. CEP: 40290-000. Tel.: (71) 3276.8200. E-mail: isabel.salviano@gmail.com
} 
conceptos subyacentes a cada dominio. A través de sus propiedades psicométricas, el instrumento demostró que tiene representatividad acerca de la percepción de los afectados por RD con respecto a su acceso a la salud y al apoyo social.

Descriptores: enfermedades raras, apoyo social, acceso a los servicios de salud, estudios de validación

\section{Introdução}

A definição das doenças raras (DR) é diferenciada em cada país. No contexto mundial, a prevalência varia entre 0,5 e 7 por 10 mil e, no Brasil, está em torno de 6,5 por 10 mil habitantes. Estima-se que existam mais de 7 mil DR no mundo (Associação da Indústria Farmacêutica de Pesquisa, 2013). Este número é variável devido à introdução constante de novas doenças nos sistemas de classificação (Luz, Silva, \& DeMontigny, 2015). Afetam entre 420 milhões e 560 milhões de pessoas no mundo e, no Brasil, 13 milhões de pessoas, aproximadamente, sofrem com as DR, traduzindo-se em cerca de $6 \%$ a $8 \%$ da população brasileira (Associação da Indústria Farmacêutica de Pesquisa, 2013). São doenças que muitas vezes se tornam crônicas, degenerativas, debilitantes e diminuem a expectativa de vida (Boy \& Schramm, 2009).

A Política Nacional de Atenção Integral às Pessoas com DR, publicada em 2014, não tem garantido o direito à saúde específico. Esses indivíduos e famílias, usuários do Sistema Único de Saúde (SUS), cursam "uma verdadeira peregrinação" em busca de atendimento, que nem sempre é satisfatório. Existe uma dificuldade em concluir o diagnóstico, pois o conhecimento das equipes de saúde sobre as DR ainda é escasso. A incerteza de quais comprometimentos a doença trará causa sentimentos de angústia e desesperança (Luz et al., 2015). Sofrimento humano, desconhecimento sobre sua condição e acesso inadequado à assistência tornam o acometido de DR especialmente dependente de sua rede de suporte social.

Estudos e debates têm sido cada vez mais comuns na tentativa de definir o que venha a ser o suporte social, conceitos, papéis, abrangência e como o apoio social repercute no bem-estar físico, mental e em possíveis mecanismos que ligam o apoio social à saúde (Franco Júnior, Heleno, \& Lopes, 2010 , Canesqui \& Barsaglini, 2012). O apoio social é relacionado à saúde desde 1976, por Cobb, que o definia como a informação que conduz o sujeito a acreditar que é cuidado, estimado e pertencente a uma rede social com obrigações mútuas, acarretando um caráter protetor para esses indivíduos (Cobb, 1976).

O uso de instrumentos subjetivos que captam a doença a partir do ponto de vista do paciente já é uma prática cada vez mais presente no cotidiano dos profissionais de saúde (Netto, Dias, Goyanna, 2016). Dessa forma, um instrumento específico para a avaliação do suporte social e acesso à saúde pode facilitar a percepção acerca do contexto em que vivem os indivíduos com DR e poderá auxiliar sobremaneira os profissionais que trabalham nos serviços de saúde no atendimento dessa população, fortalecendo a caminhada e estimulando melhorias na assistência desses indivíduos, especialmente quando acometidos por DR. Ainda que escalas voltadas para o suporte social de forma geral já tenham sido propostas, como a Escala de Satisfação com o Suporte Social (ESSS) (Ribeiro, 1999), a Medical Outcomes Study (MOS) (Griep, Chor, Faerstein, Werneck, \& Lopes, 2005), a Medical Outcomes Study Social Suporte Survey (MOS_SSS) (Fachado, Martinez, Villalva, \& Pereira, 2007) e a Escala de Redes Sociais de Lubben (LSNS) (Ribeiro et al., 2012), nenhum instrumento específico está consolidado ou amplamente aceito para essa finalidade em conjunto com acesso à saúde em 
DR. Portanto o objetivo deste estudo foi construir e validar um instrumento que possibilite avaliar a percepção do acesso à saúde e ao suporte social destinado aos acometidos por DR.

\section{Métodos}

Utilizou-se o modelo proposto por Pasquali (Pasquali, 2013), abrangendo os processos teórico, empírico e analítico para a construção, análise do conteúdo, análise semântica e análise do constructo com a finalidade de mostrar evidências preliminares de validação.

Para elaboração do instrumento, realizou-se um estudo nas principais bases de dados sobre o tema. As bases utilizadas foram: Medline, Lilacs e Banco de Dados em Enfermagem (Bdenf). Nessa etapa, foram destacadas as características comuns às diversas DR, o itinerário terapêutico e o apoio social. Além disso, o grupo de pesquisadores foi inserido nos principais centros que tratam os acometidos pelas DR.

Após leitura de artigos e vivência no cotidiano com os acometidos pelas DR, definiu-se um primeiro esboço dos domínios que trataram sobre o percurso cursado por esses sujeitos, desde o diagnóstico até o acesso ao tratamento, assim como o contexto social no qual estão inseridos. Em seguida, para criação dos itens/questões da escala, foram utilizados os critérios propostos por Pasquali (Pasquali, 2013).

A primeira etapa da validade de conteúdo ocorreu por meio da apresentação do esboço do instrumento a um grupo de especialistas. Esse grupo foi composto por sete profissionais da saúde que atuam diretamente com os acometidos por DR, na cidade de Salvador, sendo eles: um ortopedista, um endocrinologista, dois psicólogos, um assistente social, um enfermeiro e uma nutricionista. Nessa etapa, foi utilizada a metodologia Delphi (Wright \& Giovinazzo, 2000) para definição dos domínios e a construção das questões da primeira versão por meio de discussões em grupo e individualmente, presenciais e on-line, resultando em um instrumento com quatro domínios e 49 questões no modelo de escala Likert, com cinco opções de escolha.

A segunda etapa foi composta por profissionais do Brasil que atuam diretamente no Acesso à Saúde e ao Suporte Social, escolhidos por meio da análise de currículos na base de dados do Conselho Nacional de Desenvolvimento Científico (CNPq). Foram selecionados dez doutores de cada uma das seguintes áreas: enfermagem, psicologia, medicina, sociologia, serviço social, medicina preventiva, epidemiologia e saúde pública/coletiva. Assim, totalizaram-se 80 profissionais convidados. No entanto apenas 20 responderam ao convite realizado via correio eletrônico, sete recusando a avaliação e 13 aceitando o convite. Apenas três especialistas ajuizaram o instrumento de forma satisfatória, resultando em uma avaliação qualitativa com sugestões de melhoria do texto. Em seguida, o instrumento foi reavaliado pela metodologia Delphi com os especialistas locais para que as opiniões e sugestões dos especialistas do Brasil fossem consideradas.

Na Bahia, existe a Associação Baiana de Amigos da Mucopolissacaridose e outras Doenças Raras (ABAMPS), que dá suporte aos acometidos por DR na busca do acesso à saúde e à inclusão social. Com um total de 300 cadastrados, abrange todo o estado da Bahia. O tema da pesquisa foi incluído em palestras e debates realizados pela Associação, momento que propiciou a apresentação do instrumento para dois acometidos por acromegalia e a três familiares de acometidos por mucopolissacaridose, com a finalidade de realizar a análise 
semântica (pré-teste). Esses responderam os itens e posteriormente foram entrevistados quanto à compreensão de cada item em separado e do instrumento como um todo.

A validade de constructo foi realizada mediante a aplicação da escala resultante das etapas anteriores em 73 acometidos por acromegalia do Centro de Diabetes e Endocrinologia do Estado da Bahia (Cedeba). A escolha por uma população acometida por uma única patologia teve o objetivo de seguir a recomendação de uma amostra homogênea. O Cedeba apresentou uma planilha com 98 acometidos pela doença, sendo este um número significativo para realização de avaliação psicométrica. No entanto, da lista com 98 indivíduos, dois já haviam ido a óbito, dois estavam curados, um recusou a participação na pesquisa e apenas 73 foram acessados no período da coleta, a qual foi realizada entre setembro e dezembro de 2016.

Os procedimentos analíticos englobam tanto a avaliação qualitativa, utilizada para mensurar a concordância dos juízes e análise semântica, quanto à avaliação quantitativa da aplicação do instrumento construído. A avaliação qualitativa foi realizada utilizando-se a metodologia Delphi (Wright \& Giovinazzo, 2000), com grupo de especialistas (validade de conteúdo) e a análise semântica, realizada pelos acometidos por DR. Já a avaliação quantitativa da aplicação ocorreu por meio de análises das características psicométricas do instrumento, que envolveram análise fatorial exploratória (AFE) e Alfa de Cronbach.

Neste estudo, foi utilizado o programa Statistical Package for the Social Sciences (SPSS), versão 14.0 para Windows para análise dos dados. Os resultados estão apresentados por meio de tabelas, expressas em valores absolutos e percentuais - $\mathrm{n}(\%)$.

O primeiro passo da análise de dados referente à avaliação das características psicométricas do instrumento foi a aplicação do teste KMO para avaliar se os dados eram bons o suficiente para realização da análise fatorial exploratória (AFE). Em seguida, os domínios foram considerados sub-escalas independentes e a AFE foi realizada para cada um deles. Os itens que apresentaram cargas fatoriais abaixo de 0,30 foram considerados inadequados.

A confiabilidade ou consistência interna dos itens do instrumento foi testada em uma única ocasião, por meio do coeficiente Alfa de Cronbach para cada fator/domínio. Como critério complementar para decisão sobre retirada de itens, foi observado o valor do Alfa em caso de retirada do item.

O projeto foi aprovado pelo Comitê de Ética em Pesquisa da Escola Bahiana de Medicina e Saúde Pública/Fundação Bahiana para o Desenvolvimento da Ciência, CAAE: 56840516.4.0000.5544, número do parecer: 1.720.699.

\section{Resultados}

A versão inicial do instrumento, após discussão via metodologia Delphi e análise semântica pela população-meta, foi uma escala com quatro domínios, Recursos de Saúde (15 itens), Recursos Financeiros (sete itens), Aspectos Sociais (17 itens) e Aspectos Individuais (dez itens), perfazendo um total de 49 variáveis dependentes, organizadas em níveis de medida ordinal, com cinco opções de escolha, seguindo a ordem do menos favorável para o mais favorável, em dois blocos distintos: o primeiro bloco opção 1 (sempre) para opção 5 (nunca); e o segundo bloco opção 1 (não) para 5 (completamente). A análise semântica mostrou a necessidade de manter um padrão referente à organização da escrita dos itens seguindo a ordem do menos favorável para o mais favorável. 
Essa versão foi apresentada via correio eletrônico para especialistas do Brasil que trabalham com "Acesso à saúde" e "Suporte Social", escolhidos mediante consulta à base de dados do Conselho Nacional de Desenvolvimento Científico (CNPq).

Apenas três especialistas ajuizaram o instrumento, resultando em uma avaliação qualitativa com sugestões de melhoria do texto. Em seguida, o instrumento foi reavaliado pela metodologia Delphi com os especialistas locais, para que as opiniões e sugestões dos especialistas do Brasil fossem consideradas, resultando em uma nova redação de 16 itens.

O primeiro domínio avaliado foi Recursos de Saúde, com 15 itens (Tabela 1), que apresentou KMO de 0,651, permitindo a realização da AFE. Essa análise mostrou que sete dos 15 itens apresentaram uma carga fatorial abaixo de 0,30. Após a retirada desse conjunto de itens, o alfa de Cronbach foi de 0,775. A confiabilidade do alfa de Cronbach melhora ao ser retirado o item 7 do instrumento, elevando o alfa para 0,80, no entanto esse item foi mantido.

Tabela 1

Análise fatorial dos 15 itens do domínio Recursos de Saúde de 73 indivíduos acometidos por acromegalia, avaliados de setembro a dezembro de 2016, no Cedeba, Salvador, Bahia

\begin{tabular}{|c|c|c|c|}
\hline Itens & Comunalidades & $\begin{array}{l}\text { Carga } \\
\text { fatorial }\end{array}$ & $\begin{array}{l}\text { Alfa de Cronbach } \\
\text { se o item for } \\
\text { excluído }\end{array}$ \\
\hline $\begin{array}{l}8 \text { Você enfrenta dificuldades no } \\
\text { acompanhamento regular do seu tratamento? }\end{array}$ & 0,689 & 0,830 & 0,714 \\
\hline $\begin{array}{l}9 \text { Você tem dificuldade para conseguir o } \\
\text { tratamento indicado pelo médico? (exames, } \\
\text { medicamentos, procedimentos) }\end{array}$ & 0,588 & 0,767 & 0,726 \\
\hline $\begin{array}{l}4 \text { Você tem dificuldade para conseguir } \\
\text { atendimento para sua doença? }\end{array}$ & 0,482 & 0,694 & 0,736 \\
\hline $\begin{array}{l}10 \text { Você tem dificuldade para chegar até o local } \\
\text { (ou até a pessoa que administra) a medicação } \\
\text { prescrita pelo médico? }\end{array}$ & 0,476 & 0,690 & 0,742 \\
\hline $\begin{array}{l}2 \text { Você enfrenta dificuldades para realizar/ } \\
\text { agendar procedimentos como cirurgia e } \\
\text { exames no município em que reside? }\end{array}$ & 0,467 & 0,684 & 0,733 \\
\hline $\begin{array}{l}5 \text { Você precisa pagar algo do seu tratamento } \\
\text { (exames, medicamentos, procedimentos) com } \\
\text { seu próprio dinheiro? }\end{array}$ & 0,326 & 0,571 & 0,751 \\
\hline $\begin{array}{l}24 \text { De maneira geral, você está satisfeito com a } \\
\text { assistência que recebe? }\end{array}$ & 0,157 & 0,396 & 0,781 \\
\hline $\begin{array}{l}7 \text { Para ter acesso ao seu tratamento, você precisa } \\
\text { sair da sua cidade? }\end{array}$ & 0,120 & 0,346 & 0,807 \\
\hline $\begin{array}{l}22 \text { Os médicos e profissionais de saúde entendem } \\
\text { adequadamente sua doença e tratamento? }\end{array}$ & 0,068 & & \\
\hline $\begin{array}{l}20 \text { Você entendeu sobre sua doença quando } \\
\text { recebeu o diagnóstico? }\end{array}$ & 0,063 & & \\
\hline
\end{tabular}




\begin{tabular}{|c|c|c|c|}
\hline Itens & Comunalidades & $\begin{array}{c}\text { Carga } \\
\text { fatorial }\end{array}$ & $\begin{array}{l}\text { Alfa de Cronbach } \\
\text { se o item for } \\
\text { excluído }\end{array}$ \\
\hline $\begin{array}{l}6 \text { Para ter acesso (transporte, viagens, } \\
\text { hospedagens) ao seu tratamento, você precisa } \\
\text { pagar algo? }\end{array}$ & 0,053 & & \\
\hline $\begin{array}{l}3 \text { Com que frequência você precisa de } \\
\text { atendimento médico para o sua doença? }\end{array}$ & 0,040 & & \\
\hline $\begin{array}{l}23 \text { As informações que você recebe são } \\
\text { suficientes para o tratamento? }\end{array}$ & 0,031 & & \\
\hline $\begin{array}{l}21 \text { Você recebe apoio de seus familiares, amigos, } \\
\text { vizinhos no tratamento médico? }\end{array}$ & 0,015 & & \\
\hline $\begin{array}{l}1 \text { wwVocê tem dificuldade para conseguir } \\
\text { materiais de suporte (equipamentos, muletas, } \\
\text { cadeira de rodas, glicosímetro, respirador etc.) } \\
\text { de que você precisa? }\end{array}$ & 0,001 & & \\
\hline
\end{tabular}

O segundo domínio avaliado foi Recursos Financeiros, com 7 itens (Tabela 2), que apresentou KMO de 0,701, permitindo a realização da AFE. Essa análise mostrou que todos os itens apresentaram uma carga fatorial acima de 0,30 e um alfa de Cronbach de 0,748. A confiabilidade do alfa de Cronbach melhora ao retirar-se o item 29 do instrumento, elevando o alfa para 0,767 , no entanto esse item foi mantido.

Tabela 2

Análise fatorial dos sete itens do domínio Recursos Financeiros de 73 indivíduos acometidos por acromegalia, avaliados de setembro a dezembro de 2016, no Cedeba, Salvador, Bahia

\begin{tabular}{|c|c|c|c|}
\hline Itens & Comunalidades & $\begin{array}{l}\text { Carga } \\
\text { fatorial }\end{array}$ & $\begin{array}{l}\text { Alfa de Cronbach } \\
\text { se o item for } \\
\text { excluído }\end{array}$ \\
\hline $\begin{array}{l}26 \text { Você considera que a renda recebida por sua } \\
\text { família é suficiente? }\end{array}$ & 0,612 & 0,782 & 0,675 \\
\hline $\begin{array}{l}12 \text { Você precisa de ajuda dos outros para manter } \\
\text { a sua vida financeira? }\end{array}$ & 0,488 & 0,698 & 0,711 \\
\hline $\begin{array}{l}11 \text { Você precisa do apoio da comunidade, } \\
\text { associação ou familiares para pagar algo do } \\
\text { seu tratamento? }\end{array}$ & 0,484 & 0,696 & 0,704 \\
\hline $\begin{array}{l}28 \text { Você considera a casa em que mora adequada } \\
\text { para você viver com a sua doença? }\end{array}$ & 0,443 & 0,666 & 0,718 \\
\hline 27 Você contribui com a renda da sua família? & 0,372 & 0,610 & 0,719 \\
\hline 250 governo auxilia no tratamento? & 0,365 & 0,604 & 0,725 \\
\hline $\begin{array}{l}29 \text { Na cidade que você mora tem tudo de que } \\
\text { você precisa para seu tratamento? }\end{array}$ & 0,182 & 0,426 & 0,767 \\
\hline
\end{tabular}


O terceiro domínio avaliado foi Aspectos Sociais, com 17 itens (Tabela 3), que apresentou KMO de 0,781 , permitindo a realização da AFE. Essa análise mostrou que quatro dos 17 itens apresentaram uma carga fatorial abaixo de 0,30. Após a retirada desse conjunto de itens, o alfa de Cronbach foi de 0,869. A confiabilidade do alfa de Cronbach melhora ao serem retirados os itens 13 (elevando o alfa para 0,877) e 43 (elevando o alfa para 0,870), no entanto esses itens foram mantidos.

Tabela 3

Análise fatorial dos 17 itens do domínio Aspectos Sociais de 73 indivíduos acometidos por acromegalia, avaliados de setembro a dezembro de 2016, no Cedeba, Salvador, Bahia

\begin{tabular}{|c|c|c|c|}
\hline Itens & Comunalidades & $\begin{array}{l}\text { Carga } \\
\text { fatorial }\end{array}$ & $\begin{array}{l}\text { Alfa de Cronbach } \\
\text { se o item for } \\
\text { excluído }\end{array}$ \\
\hline 45 Você considera sua vida social boa? & 0,677 & 0,823 & 0,847 \\
\hline $\begin{array}{l}31 \text { Você está satisfeito com a quantidade de } \\
\text { amigos que tem? }\end{array}$ & 0,596 & 0,772 & 0,851 \\
\hline $\begin{array}{l}36 \text { Você está satisfeito com as atividades de lazer } \\
\text { que você tem? }\end{array}$ & 0,588 & 0,767 & 0,847 \\
\hline 37 Você tem apoio dos seus amigos? & 0,578 & 0,761 & 0,851 \\
\hline 38 Você tem apoio dos seus vizinhos? & 0,459 & 0,678 & 0,857 \\
\hline $\begin{array}{l}33 \text { Você gosta de ter o apoio de amigos, familiares } \\
\text { ou vizinhos para realizar suas atividades diárias? }\end{array}$ & 0,450 & 0,671 & 0,858 \\
\hline $\begin{array}{l}32 \text { Você considera importante estar próximo dos } \\
\text { amigos, vizinhos ou colegas em geral? }\end{array}$ & 0,371 & 0,609 & 0,862 \\
\hline $\begin{array}{l}42 \text { Você permite que os amigos e familiares o } \\
\text { apoiem? }\end{array}$ & 0,364 & 0,603 & 0,861 \\
\hline 35 Você gosta de sair de casa? & 0,346 & 0,588 & 0,859 \\
\hline $\begin{array}{l}40 \text { Você sai de casa para outras atividades que não } \\
\text { sejam relacionadas ao seu tratamento? }\end{array}$ & 0,338 & 0,581 & 0,862 \\
\hline 41 Você se sente feliz ao estar com outras pessoas? & 0,253 & 0,503 & 0,864 \\
\hline $\begin{array}{l}43 \text { Você acredita que a fé ou religião ajudam a } \\
\text { superar as dificuldades com sua doença? }\end{array}$ & 0,174 & 0,417 & 0,870 \\
\hline $\begin{array}{l}13 \text { Sua doença afeta sua vida social e sua relação } \\
\text { com amigos e familiares? }\end{array}$ & 0,148 & 0,385 & 0,877 \\
\hline $\begin{array}{l}39 \text { Os familiares que não moram com você ajudam } \\
\text { no seu dia a dia? }\end{array}$ & 0,069 & & \\
\hline $\begin{array}{l}44 \text { Frequentar uma igreja, templo, sinagoga, } \\
\text { comunidade ou instituição religiosa é } \\
\text { importante para você? }\end{array}$ & 0,058 & & \\
\hline $\begin{array}{l}34 \text { As associações voltadas para a sua doença são } \\
\text { importantes para você? }\end{array}$ & 0,014 & & \\
\hline $\begin{array}{l}30 \text { Você considera importante o trabalho ou os } \\
\text { estudos? }\end{array}$ & 0,010 & & \\
\hline
\end{tabular}


O quarto domínio avaliado foi Aspectos Individuais, com dez itens (Tabela 4), que apresentou KMO de 0,844, permitindo a realização da AFE. Essa análise mostrou que todos os itens apresentaram uma carga fatorial acima de 0,30 e um alfa de Cronbach de 0,891. A confiabilidade do alfa de Cronbach melhora ao retirar-se o item 16 do instrumento, elevando o alfa para 0,896, no entanto esse item foi mantido.

Tabela 4

Análise fatorial dos dez itens do domínio Aspectos Individuais de 73 indivíduos acometidos por acromegalia, avaliados de setembro a dezembro de 2016, no Cedeba, Salvador, Bahia

\begin{tabular}{|c|c|c|c|}
\hline Itens & Comunalidades & $\begin{array}{c}\text { Carga } \\
\text { fatorial }\end{array}$ & $\begin{array}{l}\text { Alfa de Cronbach se } \\
\text { o item for excluído }\end{array}$ \\
\hline 18 Você se sente sozinho? & 0,697 & 0,835 & 0,871 \\
\hline 19 Você se sente triste no seu dia a dia? & 0,664 & 0,815 & 0,873 \\
\hline 17 Você se sente angustiado? & 0,624 & 0,790 & 0,875 \\
\hline $\begin{array}{l}48 \text { Você se sente capaz de realizar as mesmas } \\
\text { atividades que outras pessoas da sua idade? }\end{array}$ & 0,618 & 0,786 & 0,874 \\
\hline $\begin{array}{l}49 \text { Você está satisfeito com sua capacidade de } \\
\text { realizar as atividades do seu dia a dia? }\end{array}$ & 0,567 & 0,753 & 0,877 \\
\hline $\begin{array}{l}15 \text { Você precisa de ajuda para suas atividades } \\
\text { diárias? }\end{array}$ & 0,474 & 0,688 & 0,881 \\
\hline $\begin{array}{l}47 \text { Você considera estar adaptado a sua } \\
\text { doença? }\end{array}$ & 0,460 & 0,678 & 0,884 \\
\hline $\begin{array}{l}14 \text { Com que frequência a sua doença impede } \\
\text { você de fazer atividades diárias? }\end{array}$ & 0,427 & 0,654 & 0,882 \\
\hline $\begin{array}{l}46 \text { De maneira geral, você está satisfeito com } \\
\text { sua vida? }\end{array}$ & 0,400 & 0,632 & 0,886 \\
\hline $\begin{array}{l}16 \text { A dor secundária a sua doença atrapalha } \\
\text { suas atividades diárias? }\end{array}$ & 0,231 & 0,480 & 0,896 \\
\hline
\end{tabular}

O resultado final após análise fatorial foi uma escala intitulada Escala de Percepção do Acesso à Saúde e Suporte Social em Acometidos por Doenças Raras (Passs), com 38 itens (Tabela 5):

- Oito itens $(1,2,3,4,5,6,7,17)$ pertencentes ao domínio Recursos de Saúde (53\% de retenção);

- Sete itens $(8,9,18,19,20,21,22)$ pertencentes ao domínio Recursos Financeiros $(100 \%$ de retenção);

- Treze itens $(10,23,24,25,26,27,28,29,30,31,32,33,34)$ pertencentes ao domínio Aspectos Sociais (76,5\% de retenção);

- Dez itens $(11,12,13,14,15,16,35,36,37,38)$ pertencentes ao domínio Aspectos Individuais (100\% de retenção). 
Tabela 5

Escala de Percepção do Acesso à Saúde e ao Suporte Social em Acometidos por Doenças Raras (Passs)

\begin{tabular}{|c|c|c|c|c|c|}
\hline Bloco 1 & Sempre & $\begin{array}{c}\text { Quase } \\
\text { sempre }\end{array}$ & Frequentemente & $\begin{array}{c}\text { Algumas } \\
\text { vezes }\end{array}$ & Nunca \\
\hline $\begin{array}{l}\text { Circule o número que está abaixo } \\
\text { da palavra que mais se aproxima da } \\
\text { percepção do entrevistado (o que } \\
\text { ele sente, vê, acredita) diante da } \\
\text { pergunta ou afirmação realizada }\end{array}$ & $\begin{array}{l}\text { Todas as } \\
\text { vezes, } \\
\text { todo } \\
\text { tempo }\end{array}$ & $\begin{array}{l}\text { A maior } \\
\text { parte das } \\
\text { vezes }\end{array}$ & $\begin{array}{c}\text { Nem sempre } \\
\text { nem nunca, mais } \\
\text { ou menos }\end{array}$ & $\begin{array}{l}\text { Poucas } \\
\text { vezes, } \\
\text { quase } \\
\text { nunca }\end{array}$ & $\begin{array}{c}\text { De nenhuma } \\
\text { forma, em } \\
\text { nenhum } \\
\text { momento }\end{array}$ \\
\hline
\end{tabular}

1 Você enfrenta dificuldades para realizar/agendar procedimentos como cirurgia e exames no municí1 pio em que reside?

2 Você tem dificuldade para conseguir atendimento para sua doença?

3 Você precisa pagar algo do seu tratamento (exames, medicamentos, procedimentos) com seu próprio dinheiro?

4 Para ter acesso ao seu tratamento, você precisa sair da sua cidade?

5 Você enfrenta dificuldades no acompanhamento regular do seu tratamento?

6 Você tem dificuldade para conseguir o tratamento indicado pelo médico? (exames, medicamentos, procedimentos)

7 Você tem dificuldade para chegar até o local (ou até a pessoa que administra) a medicação prescrita pelo médico?

8 Você precisa do apoio da comunidade, associação ou familiares para pagar algo do seu tratamento?

9 Você precisa de ajuda dos outros para manter a sua vida financeira?

10 Sua doença afeta sua vida social e sua relação com amigos e familiares?

11 Com que frequência a sua doença impede você de fazer atividades diárias?

12 Você precisa de ajuda para suas atividades diárias?

13 A dor secundária a sua doença atrapalha suas atividades diárias?

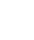
4 Você se sente angustiado?

15 Você se sente sozinho?

16 Você se sente triste no seu dia a dia?

$\begin{array}{lllll}1 & 2 & 3 & 4 & 5 \\ 1 & 2 & 3 & 4 & 5 \\ 1 & 2 & 3 & 4 & 5 \\ 1 & 2 & 3 & \\ 1 & 2 & 3 & 5 \\ 1 & 2 & 3 & 4 & 5 \\ 1 & 2 & 3 & 4 & 5 \\ 1 & 2 & 3 & 4 & 5 \\ 1 & 2 & 3 & 4 & 5\end{array}$

\section{5}

5




\begin{tabular}{|c|c|c|c|c|c|}
\hline Bloco 2 & Não & Pouco & Moderadamente & Muito & Completamente \\
\hline $\begin{array}{l}\text { Circule o número que está abaixo } \\
\text { da palavra que mais se aproxima da } \\
\text { percepção do entrevistado (o que ele } \\
\text { sente, vê, acredita) diante da pergun- } \\
\text { ta ou afirmação realizada }\end{array}$ & $\begin{array}{l}\text { Nada, } \\
\text { de jeito } \\
\text { nenhum }\end{array}$ & $\begin{array}{l}\text { Levemente, } \\
\text { quase nada }\end{array}$ & $\begin{array}{l}\text { Mais ou me- } \\
\text { nos, nem tanto } \\
\text { nem tão pouco, } \\
\text { metade }\end{array}$ & $\begin{array}{l}\text { Bastante, } \\
\text { mais do } \\
\text { que a } \\
\text { metade }\end{array}$ & $\begin{array}{l}\text { Extremamente, } \\
\text { imensamente }\end{array}$ \\
\hline $\begin{array}{l}17 \text { De maneira geral, você está } \\
\text { satisfeito com a assistência que } \\
\text { recebe? }\end{array}$ & 1 & 2 & 3 & 4 & 5 \\
\hline 18 O governo auxilia no tratamento? & 1 & 2 & 3 & 4 & 5 \\
\hline $\begin{array}{l}19 \text { Você considera que a renda rece- } \\
\text { bida por sua família é suficiente? }\end{array}$ & 1 & 2 & 3 & 4 & 5 \\
\hline $\begin{array}{l}20 \text { Você contribui com a renda da } \\
\text { sua família? }\end{array}$ & 1 & 2 & 3 & 4 & 5 \\
\hline $\begin{array}{l}21 \text { Você considera a casa em que } \\
\text { mora adequada para você viver } \\
\text { com a sua doença? }\end{array}$ & 1 & 2 & 3 & 4 & 5 \\
\hline $\begin{array}{l}22 \text { Na cidade em que você mora tem } \\
\text { tudo de que você precisa para } \\
\text { seu tratamento? }\end{array}$ & 1 & 2 & 3 & 4 & 5 \\
\hline $\begin{array}{l}23 \text { Você está satisfeito com a quanti- } \\
\text { dade de amigos que tem? }\end{array}$ & 1 & 2 & 3 & 4 & 5 \\
\hline $\begin{array}{l}24 \text { Você considera importante estar } \\
\text { próximo dos amigos, vizinhos ou } \\
\text { colegas em geral? }\end{array}$ & 1 & 2 & 3 & 4 & 5 \\
\hline $\begin{array}{l}25 \text { Você gosta de ter o apoio de ami- } \\
\text { gos, familiares ou vizinhos para } \\
\text { realizar suas atividades diárias? }\end{array}$ & 1 & 2 & 3 & 4 & 5 \\
\hline 26 Você gosta de sair de casa? & 1 & 2 & 3 & 4 & 5 \\
\hline $\begin{array}{l}27 \text { Você está satisfeito com as ativi- } \\
\text { dades de lazer que você tem? }\end{array}$ & 1 & 2 & 3 & 4 & 5 \\
\hline 28 Você tem apoio dos seus amigos? & 1 & 2 & 3 & 4 & 5 \\
\hline 29 Você tem apoio dos seus vizinhos? & 1 & 2 & 3 & 4 & 5 \\
\hline $\begin{array}{l}30 \text { Você sai de casa para outras ativi- } \\
\text { dades que não sejam relaciona- } \\
\text { das ao seu tratamento? }\end{array}$ & 1 & 2 & 3 & 4 & 5 \\
\hline $\begin{array}{l}31 \text { Você se sente feliz ao estar com } \\
\text { outras pessoas? }\end{array}$ & 1 & 2 & 3 & 4 & 5 \\
\hline $\begin{array}{l}32 \text { Você permite que os amigos e } \\
\text { familiares o apoiem? }\end{array}$ & 1 & 2 & 3 & 4 & 5 \\
\hline $\begin{array}{l}33 \text { Você acredita que a fé ou religião } \\
\text { ajudam a superar as dificuldades } \\
\text { com sua doença? }\end{array}$ & 1 & 2 & 3 & 4 & 5 \\
\hline $\begin{array}{l}34 \text { Você considera sua vida social } \\
\text { boa? }\end{array}$ & 1 & 2 & 3 & 4 & 5 \\
\hline $\begin{array}{l}35 \text { De maneira geral, você está satis- } \\
\text { feito com sua vida? }\end{array}$ & 1 & 2 & 3 & 4 & 5 \\
\hline $\begin{array}{l}36 \text { Você considera estar adaptado a } \\
\text { sua doença? }\end{array}$ & 1 & 2 & 3 & 4 & 5 \\
\hline $\begin{array}{l}37 \text { Você se sente capaz de realizar } \\
\text { as mesmas atividades que outras } \\
\text { pessoas da sua idade? }\end{array}$ & 1 & 2 & 3 & 4 & 5 \\
\hline $\begin{array}{l}38 \text { Você está satisfeito com sua capa- } \\
\text { cidade de realizar as atividades } \\
\text { do seu dia a dia? }\end{array}$ & 1 & 2 & 3 & 4 & 5 \\
\hline
\end{tabular}


Os resultados do instrumento são obtidos por domínios, sendo que a fórmula para o cálculo de cada domínio considera os valores referentes aos itens exclusivos do domínio. Somamse os valores resultantes das escolhas dos acometidos a cada um dos itens específicos do domínio, subtraindo a quantidade total de itens (que é o valor mínimo que se pode obter com essa somatória) seguido da divisão do resultado por um valor resultante da somatória de todos os itens, subtraído do valor mínimo dessa somatória (que é o total de itens que o domínio possui) e multiplicando o valor final por 100, resultando em uma porcentagem.

Fórmula: $\underline{X(\Sigma \text { dos valores de } 1 \text { a } 5 \text { dos itens do domínio)-quantidade total de itens }} \times 100$

( $\Sigma$ dos valores de todos os itens - a quantidade total de itens)

\section{Discussão}

O número de itens que compõem o domínio Recursos de Saúde do instrumento desenvolvido foi reduzido à metade após a condução das análises de suas propriedades psicométricas. É possível notar que os itens mantidos no domínio agrupam conteúdos que tratam de dificuldades concretas referentes ao acompanhamento regular do tratamento, dificuldades para conseguir o tratamento indicado pelo médico e dificuldades em alcançar o atendimento para a doença, configurando-se esses em alguns dos entraves comuns enfrentados pelos acometidos por DR (Horovitz, Cardoso, Llerena Junior, \& Mattos, 2006; Fonseca, 2014; Aith, F., Bujdoso, Nascimento, \& Dallari, 2014).

A satisfação com a assistência recebida, questionada em um dos itens, apresenta um tema que perpassa todo o itinerário terapêutico do acometido pela DR: vai desde a atuação dos profissionais, na tentativa de prestar uma assistência mais específica, evitando maiores complicações (Oliveira, Melo, Tashima, Pelloso, \& Silva, 2012), passando pelas relações da equipe cuidadora e com suas famílias (Luz, 2008), até as questões enfrentadas na busca por um tratamento (Boy \& Schramm, 2009; Diniz, Medeiros, \& Schwartz, 2012).

Optou-se por manter o item que questiona a necessidade de o acometido sair da sua cidade para obter acesso ao tratamento, por ser uma realidade dos moradores de cidades pequenas que não contam com Centros de Referência e precisam se deslocar para cidades maiores (Luz et al., 2015). Além da questão supracitada, o item referente às dificuldades para realizar/agendar procedimentos, como cirurgia e exames no município em que reside, condiz com as diretrizes da Atenção Integral às Pessoas com DR que centralizam na Atenção Especializada Ambulatorial e Hospitalar os procedimentos mais específicos (Brasil, 2014).

Entre os itens excluídos, os que questionaram se os médicos e profissionais de saúde entendem adequadamente sobre a doença e o tratamento e se as informações recebidas são suficientes para o tratamento não apresentaram uma carga fatorial satisfatória, mesmo sabendo-se que existe uma dificuldade por parte dos profissionais na compreensão das DR (Luz et al. 2015; Moura, 2013). A baixa carga fatorial referente a essas questões pode ter ocorrido por se tratar de aspectos muito subjetivos, talvez esses itens necessitem de um aprimoramento ou até mesmo um desdobramento em mais questões para que assim exista uma possibilidade mais concreta de avaliação, tratando-se de uma limitação deste estudo.

É provável, portanto, que o fato de os itens mencionarem aspectos tão distintos, no que se refere ao acesso à saúde, tenha sido o responsável pela necessidade de exclusão de um grande 
número de indicadores. Uma proposta para a melhoria desse domínio talvez seja o seu desdobramento em dois, na tentativa de abarcar um número maior de itens que cubram mais aspectos referentes aos recursos de saúde dos acometidos por DR. Considera-se, contudo, que os itens retidos são capazes de representar de forma satisfatória importantes facetas dos Recursos de Saúde, como: dificuldades para acesso, atendimento, agendamento de procedimentos, acompanhamento regular do tratamento, além de uma insatisfação com o atendimento recebido.

O domínio Recursos Financeiros manteve o número de itens inalterado após a análise fatorial, reunindo questões que fazem referência ao contexto financeiro individual e familiar dos acometidos. Ao ser retirado o item referente a ter na cidade em que reside tudo que precisa para o tratamento, o alfa de Cronbach apresenta discreta melhora, no entanto ele foi mantido por retratar mais uma vez as dificuldades em ter próximo ao acometido os recursos entendidos como necessários para sua terapêutica.

Esse domínio identificou em seus itens a importância de ter uma renda suficiente ou um auxílio do governo para ajudar na terapêutica, assim como nas necessidades de contribuição financeira por parte de outras pessoas e da comunidade para manter a vida financeira ou pagar algo do tratamento. A dimensão desse domínio em DR vai muito além da compra de medicações, que, no caso dos medicamentos órfãos, são bastante onerosos, perpassando a possibilidade de ter uma condição de acesso aos bens que interferem na qualidade de vida, os considerados determinantes e condicionantes da saúde, como lazer, moradia, alimentação, saneamento básico, transporte e educação (Brasil, 2009).

A DR traz prejuízos financeiros importantes no sistema familiar, elevando o risco quando as famílias residem distante dos serviços de referência. Assim, o local de residência pode atenuar as problemáticas que aparecem na trajetória do acesso aos serviços de saúde. Nesse sentido, os cuidados não devem ser direcionados apenas para a DR, e sim para uma organização da família que, na maioria das vezes, precisa acompanhar o acometido para o tratamento.

O número de itens que compõem o domínio Aspectos Sociais foi pouco alterado, de 17 para 13. Mantiveram-se questões que envolvem claramente o contexto social dos acometidos, como aqueles referentes a: satisfação com a quantidade de amigos; apoio de amigos e vizinhos; apoio de amigos, familiares e vizinhos nas atividades diárias e permissão que esses os apoiem. Famílias de crianças com DR afirmam a importância de familiares e amigos nos cuidados diários, o que gera tranquilidade e segurança, contribuindo inclusive para a manutenção da unidade familiar (Luz et al., 2016).

Alguns itens, como o referente a familiares que não moram no mesmo lar do acometido e o ajudam no dia a dia, e o relativo ao fato de a doença afetar a vida social e a relação com amigos e familiares, de forma geral, tinham semelhanças com os itens supracitados e foram retirados, demonstrando a importância na construção de instrumentos, com o cuidado atinente à variabilidade de questões que mostram evidências similares (Souza, 2007; Coluci et al., 2015).

O item voltado à importância que o acometido atribui ao fato de estar próximo dos amigos, vizinhos e colegas se assemelha a um dado apresentado por um estudo português, o qual mostrou a importância da satisfação com o suporte social em um grupo de pacientes que realizaram transplante hepático: eles afirmaram a importância da presença de amigos, principalmente nos momentos de maior dificuldade, influenciando na recuperação, por se sentirem amados e valorizados (Abrunheiro, 2005). 
As relações sociais podem se modificar com o aparecimento de uma DR, visto que as preocupações com o tratamento acabam sendo a prioridade e, por vezes, impedem que o ambiente natural da família permaneça como antes. No entanto algumas famílias de acometidos por DR referem à necessidade do convívio com outras pessoas, desde pessoas com histórias de vida parecida, favorecendo o compartilhamento de experiências, até outras fora do contexto das DR, amenizando o estigma e o preconceito social que os qualificam como frágeis (Luz et al., 2016). Logo, percebe-se a importância de itens do instrumento referentes a gostar de sair de casa, sair de casa para atividades que não sejam relacionadas ao tratamento e se sentir feliz ao estar com outras pessoas.

Todos os itens que foram retidos se voltam para o conceito de que o apoio social se relaciona às ações e às relações das pessoas entre si, em determinadas situações ou contextos, movido pelas tradições, crenças e conhecimentos de experiências cotidianas, de modo que o sujeito perceba o apoio social de acordo com as particularidades da personalidade e os modos de enfrentamento dos episódios de vida, da estabilidade social e das redes a que pertencem (Canesqui \& Barsaglini, 2012).

Não houve alteração no número de itens do domínio Aspectos Individuais, permanecendo com os dez itens sugeridos pela versão submetida à análise fatorial. As questões desse domínio se voltam para a individualidade do acometido pela DR, abordando sentimentos como solidão, tristeza e angústia, sendo essas emoções expressadas por acometidos por DR e seus familiares (Luz, 2014; 2008; Pelentsov, Laws, \& Esterman, 2015).

Esse domínio traz questões que variam de acordo com a DR que o paciente venha ter, como os itens referentes a: frequência que a doença impede a realização de atividades diárias, precisar de ajuda nas atividades diárias e capacidade de realizar atividades diárias da mesma forma que outras pessoas com a mesma idade. Todas essas questões se voltam para a possibilidade e disposição para a realização das atividades do dia a dia, e uma DR com manifestações físicas importantes, como a MPS (Scarpa et al., 2011), ou que promova perdas progressivas de habilidades, como a esclerose lateral amiotrófica (ELA) (Jeppesen, Rahbek, Greda, \& Hansen, 2015), poderia apresentar respostas similares, cabendo ao profissional que estiver utilizando o instrumento interpretar e utilizar os dados dessa percepção de forma individualizada e direcionada à melhoria da capacidade referente às atividades corriqueiras.

O instrumento final desenvolvido reuniu evidências de validade, revelando qualidade dos itens em representar os conceitos subjacentes a cada domínio. Por meio de suas propriedades psicométricas, o instrumento mostrou que tem representatividade no que concerne à percepção do acometido por DR quanto ao seu acesso à saúde e ao suporte social. Espera-se que o instrumento desenvolvido atue como estratégia de valorização e melhoria da Política Nacional de Atenção Integral às Pessoas com Doenças Raras. Trabalhos como este poderão servir de base para o planejamento, implantação e revisão da Política voltada às necessidades desses usuários.

\section{Referências}

Abrunheiro, L. M. M. (2005). A satisfação com o suporte social e a qualidade de vida no doente após transplante hepático. Psicologia.com.pt., 1-15.

Aith, F., Bujdoso, Y., Nascimento, P. R., Dallari \& S. G.( 2014). Os princípios da universalidade e integralidade do SUS sob a perspectiva da política de doenças raras e da incorporação 
tecnológica. Revista Direito Sanitário, 15(1),10-39. doi:10.11606/issn.2316-9044. v15i1p10-39.

Associação da Indústria Farmacêutica de Pesquisa. (2013). Doenças raras: Contribuições para uma política nacional. São Paulo: [s.n.].

Boy, R. \& Schramm, F. R. (2009). Bioética da proteção e tratamento de doenças genéticas raras no Brasil: o caso das doenças de depósito lisossomal. Cadernos de Saúde Pública, 25(6), 1276-1284. doi:10.1590/\$0102-311X2009000600010.

Brasil. Ministério da Saúde. (2009). I Seminário sobre a Política Nacional de Promoção da Saúde. Série D. Brasília, DF.

Brasil. Ministério da Saúde. (2014). Diretrizes para atenção integral às pessoas com doenças raras no Sistema Único de Saúde - SUS. Brasília, DF.

Canesqui, A. M. \& Barsaglini, R. A. (2012). Apoio social e saúde: Pontos de vista das ciências sociais e humanas. Ciência e Saúde Coletiva, 17(5), 1103-1114. doi:10.1590/ S1413-81232012000500002.

Cobb, S. (1976). Social support as a moderator of life stress. Psychosom Med, 38(5), 300-314. DOI:10.1097/00006842-197609000-00003

Coluci, M. Z. O., Alexandre, N. M. C., \& Milani, D. (2015).Construção de instrumentos de medida na área da saúde. Ciência \& Saúde Coletiva, 20(3),925-936. doi:10.1590/1413-81232015203.04332013

Diniz, D., Medeiros, M., \& Schwartz, I. V. D. (2012). Consequências da judicialização das políticas de saúde: Custos de medicamentos para as mucopolissacaridoses. Cadernos de Saúde Pública, 28(3), 479-489. doi:10.1590/S0102-311X2012000300008.

Fachado, A. A., Martinez, M. A., Villalva, M. C., \& Pereira, M. G. (2007). Adaptação cultural e validação da versão portuguesa do questionário Medical Outcomes Study Social Support Survey (MOS-SSS). Acta Médica Portuguesa, 20(6), 525-534.

Fonseca, R.V. G. (2014). A construção de uma política pública para doenças raras no Brasil (Trabalho de conclusão de curso, Universidade de Brasília, Brasília, DF, Brasil).

Franco Júnior, A. J. A., Heleno, M. G. V., \& Lopes, A. P. (2010). Qualidade de vida e controle glicêmico do paciente portador de Diabetes Mellitus tipo 2. Revista Psicologia e Saúde, 5(2), 102-108.

Griep, R. H., Chor, D., Faerstein, E., Werneck, G. L., \& Lopes, C. S.( 2005). Construct validity of the Medical Outcomes Study's social support scale adapted to Portuguese in the Pró-Saúde Study. Cadernos de Saúde Pública, 21(3),703-714. doi:10.1590/ S0102-311X2005000300004

Horovitz, D. D. G., Cardoso, M. H. C. A., Llerena Junior, J. C., \& Mattos, R. A. (2006). Atenção aos defeitos congênitos no Brasil: Características do atendimento e propostas para formulação de políticas públicas em genética clínica. Cadernos de Saúde Pública, 22(12), 2599-2609. doi:10.1590/S0102-311X2006001200010

Jeppesen, J., Rahbek, J., Greda, L. O., \& Hansen, H. P. (2015). How Narrative Journalistic Stories Can Communicate the Individual's Challenges of Daily Living with Amyotrophic Lateral Sclerosis. Patient, 8(1), 41-49. doi:10.1007/s40271-014-0088-6.

Luz, G. S. (2008). Fibrose Cística: des-velando o significado para a família (Dissertação de mestrado, Universidade Etadual de Maringá, Maringá, PR, Brasil). 
Luz, G. S. (2014). Relação entre famílias de pessoas com doenças raras e os serviços de saúde: Desafios e possibilidades (Tese de doutorado, Universidade Federal do Rio Grande, Rio Grande, RS, Brasil).

Luz, G. S., Silva, M. R. S., \& DeMontigny, F. (2015). Doenças raras: itinerário diagnóstico e terapêutico das famílias de pessoas afetadas. Acta Paulista de Enfermagem, 28(5), 395400. doi:10.1590/1982-0194201500067.

Luz, G. S., Silva, M. R. S., \& DeMontigny, F. (2016). Necessidades prioritárias referidas pelas famílias de pessoas com Doenças Raras. Texto \& Contexto Enfermagem, 25(4), 9. doi:10.1590/0104-07072016000590015

Moura, R. M. (2013). Intinerário diagnóstico e terapêutico das famílias e dos pacientes com doenças órfãs (Dissertação de mestrado, Universidade Estadual de Minas Gerais, Belo Horizonte, MG, Brasil).

Netto, J. J. M., Dias, M. S. A., \& Goyanna, N. F. (2016). Uso de instrumentos enquanto tecnologia para a saúde. Saúde em Redes, 2(1), 65-72. doi:10.18310/2446-4813.2016v2n11p65-72.

Oliveira, T. F. D. E., Melo, S. C. S. D., Tashima, C. M., Pelloso, S. M., \& Silva, N. M. M. G. (2012). Qualidade de vida de pacientes com acromegalia. Fiep Bulletin, 82(2), 1-7.

Pasquali, L. (2013). Psicometria: Teoria dos testes na psicologia e na educação (5a ed.). Petrópolis, RJ: Vozes.

Pelentsov, L. J., Laws, T. A., \& Esterman, A. J. (2015). The supportive care needs of parents caring for a child with a rare disease: A scoping review. Disabil and Health Journal, 8(4), 475-91. doi:10.1016/j.dhjo.2015.03.009

Ribeiro, J. L. P. (1999). Escala de satisfação com o suporte social (ESSS). Análise Psicológica, $3(\mathrm{XVII}), 547-558$.

Ribeiro, O., Teixeira, L., Duarte, N., Azevedo, M. J., Araújo, L. Barbosa S., \& Paúl, C. (2012). Versão o0 de Lubben (LSNS-6). Revista Temática Kairós Gerontologia, 15(1), 217-34.

Scarpa, M., Almássy, Z., Beck, M., Bodamer, O., Bruce, I. A., De Meirleir, L., . . . Wraith, J. E. (2011). Hunter Syndrome Europena Expert Council. Mucopolysaccharidosis type II: European recommendations for the diagnosis and multidisciplinary management of a rare disease. Orphanet Journal of Rare Diseases, 6(72), 2-18. doi:10.1186/1750-1172-6-72.

Souza, I. (2007). Elaboração e validação de instrumentos de pesquisa. Núcleo de Estudos em Comportamento Organizacional, Universidade Federal Rural do Rio de Janeiro. Seropédica: UFRRJ.

Wright, J., Giovinazzo, R. (2000). Delphi: uma ferramenta de apoio ao planejamento prospectivo. Cadernos de Pesquisas em Administração, 1(12), 54-65.

Recebido: 29/07/2019

Última revisão: 12/09/2019

Aceite final: 11/02/2020

\section{Sobre os autores:}

Isabel Cristina de Barros Salviano: Mestre em Tecnologias. Professora de Práticas em Enfermagem na Universidade Salgado de Oliveira, Salvador, Bahia, Brasil. E-mail: isabel.salviano@gmail.com, Orcid: https://orcid.org/0000-0001-5603-4136 
Martha Moreira Cavalcante Castro: Doutora em Medicina e Saúde. Professora adjunta IV, pesquisadora efundadora do Centro de Dorna Universidade Federal da Bahia, Departamento de Fisioterapia, Salvador, Bahia, Brasil. E-mail: marthamccastro@gmail.com, Orcid: https://orcid.org/0000-0002-7868-1120

Marcos Antônio Almeida Matos: Doutor em Ortopedia e Traumatologia. Coordenador do curso de Mestrado em Tecnologias em Saúde na Escola Bahiana de Medicina e Saúde Pública, Salvador, Bahia, Brasil. E-mail: marcos.almeida@hotmail.com, Orcid: https://orcid.org/0000-0002-3592-986X

Carolina Villa Nova Aguiar: Doutora em Psicologia. Professora adjunta do curso de Psicologia na Escola Bahiana de Medicina e Saúde Pública, Salvador, Bahia, Brasil. E-mail: carol.vna@gmail.com, Orcid: https://orcid.org/0000-0002-8213-3100 